\title{
Auditorías PCAOB: Mejores estrategias para cumplir con el marco normativo del PCAOB en revisiones de control interno (PCAOB audits: Best audit strategies to comply with the regulatory framework of the PCAOB on internal control reviews)
}

\section{' Carlos Daniel Barrientos Elizondo \\ ii Fernando Iram Lozano Guerra}

\begin{abstract}
This article is regarding the regulatory framework of internal control applicable to public companies in the United States, and about which are the official requirements stablished by the authority, for the reviews performed by external auditors, in order to expose which are some audit strategies to apply to meet the regulatory framework and to streamline the work at audit firms, with the purpose of propitiate a significant positive impact on the time and resources invested in the audit.
\end{abstract}

Key words: audit, audit strategies, internal control, PCAOB audits.

JEL: M420, M480.

Resumen. Este artículo es referente al marco normativo de control interno aplicable para empresas que son públicas en Estados Unidos y acerca de cuáles son los requerimientos oficiales establecidos por la autoridad para la revisión de dichas compañías por parte de los auditores externos, con el objetivo de exponer cuáles son algunas estrategias de auditoría a aplicar para cumplir el marco normativo y poder eficientar el trabajo realizado por las firmas de auditoría con el objetivo de tener un impacto significativo positivo, en el tiempo y con los recursos invertidos en la revisión.

Palabras clave: auditoría, auditorías PCAOB, control interno,estrategias de auditoría.

\footnotetext{
UANL. Universidad Autónoma de Nuevo León•carlos.barrientos.elizondo@gmail.com

ii UANL. Universidad Autónoma de Nuevo León•flozanoguerra@hotmail.com
} 


\section{Introducción}

En el año 2002 se marcó un cambio radical en la forma en que los auditores realizaban su trabajo y emitían sus opiniones profesionales (Brickey, 2003; Li, 2010). Después de escándalos financieros como el de "Enron Corporation" a finales de 2001 y el caso "WorldCom" a finales de 2002, se vislumbraron grandes deficiencias en el trabajo de las firmas de auditoría para dar confianza en la información financiera de sus clientes.

La Comisión Nacional de Valores de los Estados Unidos (SEC) y el gobierno federal de los Estados Unidos, preocupados por la falta de confiabilidad en la información que las compañías públicas proporcionan a sus inversionistas, y después de encontrar terribles ineficiencias por parte de reconocidas firmas de auditoría, decidieron tomar cartas en al asunto para devolverle a los inversionistas, actuales y potenciales, verdadera confianza en el mercado de valores de la unión americana (Fornelli, 2015).

Por todo la anterior, el Congreso de los Estados Unidos aprobó la Ley Sarbanes-Oxley en 2002, creando el PCAOB (por sus siglas en inglés, que significan Public Company Accounting Oversight Board), órgano encargado de revisar el trabajo de auditoría de las diversas firmas que tengan relación con todas aquellas empresas públicas en Estados Unidos (Nagy, 2005).

A partir de su creación, el PCAOB ha establecido diversas normas enfocadas en delimitar las responsabilidades de los auditores en cuanto a estándares mínimos de calidad que deben considerarse para sus revisiones, y obligaciones que deben cumplir las compañías públicas para asegurar que sus políticas y procedimientos, referentes al control interno, aseguren la emisión confiable de información financiera.

Considerando todo lo anterior, las firmas de auditoría buscan adaptar sus procedimientos para cumplir con los estatutos marcados por el PCAOB, pero con la misión de ser cada vez más eficientes, disminuyendo el tiempo y los costos, pero cumpliendo con los estándares de calidad establecidos.

\section{Metodología}

Este artículo se desarrolló utilizando fuentes teóricas de información y bases de datos académicas de revistas especializadas: Google Scholar, Conricyt, ISI Way of Knowledge, entre otras; con ellas se construyó un cuerpo de conocimiento 
que versa sobre el marco normativo que deben de aplicar los auditores en sus revisiones y cuáles son algunas estrategias de auditoría que pueden eficientar este tipo de trabajos.

Se utilizaron bases de datos de fuentes secundarias de órganos institucionales como, por ejemplo: COSO (por sus siglas en inglés que significan The Committee of Sponsoring Organizations of the Treadway Commission), PCAOB, SEC, OCDE, entre otras; en estas se encontraron datos y series históricas sobre el tema en cuestión para analizar el efecto que han tenido tales cambios en el área de auditoría y los resultados que se han obtenido desde la implementación de este comité.

Se analizaron críticamente estos documentos para evaluar y determinar el efecto que tiene la normatividad vigente en cuanto al trabajo y responsabilidades del auditor bajo las normas del PCAOB, y se presentan tres estrategias básicas que se discutirán en el presente documento, que permitirán mejorar la eficiencia en el trabajo de auditoría durante revisiones integradas:

1. Aplicación de procedimientos para mejorar el entendimiento de la entidad.

2. Mayor utilización del trabajo de auditoría interna.

3. Mayor aplicación de procedimientos analíticos.

\section{Revisión de la literatura}

\section{Definición de empresas públicas}

Este artículo está enfocado en analizar cuáles son las mejores estrategias de auditoría que se podrían aplicar cuando existen revisiones de control interno en empresas públicas. Como primer punto es importante definir qué se entiende por empresas públicas, ya que es donde se enfoca la atención de este artículo.

De acuerdo a WebFinance Inc. (2016) una empresa pública es "una empresa que ha emitido valores a través de una oferta, y que ahora se negocian en el mercado abierto". Es decir, se entiende por empresa pública, toda aquella que cotice en un mercado de valores, reconocido oficialmente como tal.

Es en estos mercados de valores donde oferentes y demandantes de dinero o insumos negocian ante el público inversionista sus propuestas, y con ello obtienen u ofrecen recursos que, de acuerdo a su conveniencia, les sean más favorables. 
Estas compañías, para poder enlistarse y participar en este tipo de mercados, deben de cumplir con los estándares establecidos por los organismos reguladores correspondientes al mercado donde se esté inscrito.

Como en este artículo nos enfocaremos en las empresas públicas de los Estados Unidos, se hablará principalmente de las empresas que cotizan en la bolsa de valores de Nueva York porque debido al importante número de empresas que participan en ella y al gran volumen de operaciones que se llevan a cabo en la misma, es considerada la más grande del mundo y, por ende, una de las que más requisitos solicita para permanecer y participar en ella.

\section{Marco normativo que rige a las empresas públicas en USA.}

Después de los escándalos internacionalmente conocidos, el gobierno de Estados Unidos, liderado por los congresistas de los distintos partidos políticos, crearon la ley conocida como Sarbanes-Oxley (Beckstead, 2002).

Esta ley, también llamada Ley SOx, SarbOx o SOA, debe su nombre al senador Paul Sarbanes y al congresista Michael G. Oxley, sus principales impulsores. La Ley Sarbanes-Oxley fue creada con el propósito de proteger los intereses de los accionistas a través de nuevas medidas regulatorias aplicables para todos los entes que participan en este mercado (Díaz Morales, 2005).

Según Díaz Morales (2005), con esta ley:

1) Las obligaciones y responsabilidades de cada uno de los que intervienen en las sociedades cotizadas en el mercado americano:

- Se hacen más explícitas;

- Se acompañan de un mayor seguimiento;

- Se penalizan de manera significativa cuando se incumplen.

La ley incluye medidas preventivas muy representativas en cuanto a la responsabilidad corporativa y la independencia de los auditores (Beckstead, 2002); su emisión implica un cambio radical en cuanto a la regulación que las empresas públicas tenían que cumplir antes de su publicación.

Según Cortijo Gallego (2007), esta ley "puede considerase como uno de los mayores desafíos de los últimos tiempos en materia de legislación empresarial ya que afecta no solo a las compañías cotizadas en los mercados financieros estadounidenses sino también a los profesionales de la contabilidad y auditoría". Los contenidos de esta ley se organizan en secciones y títulos (ver Tabla 1). 
Tabla 1. Contenido de la Ley Sarbanes-Oxley

Título I: Junta de Supervisión de Firmas de Auditoría

Título II: Independencia de los Auditores

Título III: Responsabilidad Corporativa

Título IV: Revelaciones Financieras Ampliadas y Mejoradas

Título V: Conflicto de Intereses del Analista

Título Vl: Recursos y Autoridad de la Comisión

Título VII: Estudios e Informes

Título VIII: Responsabilidad Corporativa y Fraude

Título IX: Sanciones por Crímenes de Cuello Blanco

Título X: Declaraciones por Impuestos Corporativos

Título XI: Responsabilidad por Fraudes Corporativos

Fuente: Tabla obtenida del artículo de Cortijo Gallego (2007).

Según Díaz Morales (2005) la Ley Sarbanes-Oxley es un texto cuyos contenidos principales se agrupan en seis grandes áreas que afectan a todas las sociedades cotizadas en los mercados americanos:

- Mejora en la calidad de la información pública y en los detalles de la misma.

- Reforzamiento de responsabilidades en el Gobierno Corporativo de las sociedades.

- Mejora en las conductas y comportamientos éticos exigibles: mayores exigencias de responsabilidad en los temas de gestión indebida de información confidencial.

- Aumento de la supervisión a las actuaciones en los mercados cotizados.

- Incremento del régimen sancionador asociado a incumplimientos.

- Aumento de exigencia y presión sobre la independencia efectiva de los auditores.

Junto con la creación e implementación de esta ley sobrevinieron cambios significativos donde la profesión contable y de auditoría se vio sujeta a nuevas reglas. La Ley Sarbanes-Oxley (Ley SOX o SAROX) incluyó entre sus estatutos la creación de un comité de supervisión y regulación de los auditores, desde firmas de auditoría de gran prestigio hasta auditores independientes cuyos clientes coticen en la bolsa de valores de la unión americana. Este comité es mejor conocido como el PCAOB y está dotado de toda la autoridad por parte del Gobierno Federal de los Estados Unidos para vigilar y sancionar todo acto ilegal que atente 
contra las leyes establecidas en materia del mercado de valores y de información financiera que, para efectos de compañías públicas, se solicite.

Este cambio radical implica que la actividad profesional de los auditores pasa de estar en un entorno totalmente autorregulado a ser vigilado directamente por un órgano instituido por el gobierno, con reglas muy estrictas y sanciones significativas en caso de algún incumplimiento (Reiter \& Williams, 2013)

\section{Marco normativo y organismo que rige a los auditores de empresas públicas}

La Ley Sarbanes-Oxley facultó a la SEC para la creación de un órgano regulador que supervisara la actuación y la calidad del trabajo realizado por las firmas de auditores en relación con las empresas registradas en la SEC. Surgió así el $\mathrm{PCAOB}$, institución responsable no solo de la supervisión de los auditores sino también de la emisión de normas de auditoría a las que deben sujetarse los auditores de las empresas emisoras en la SEC.

Según el mismo comité del PCAOB (2016b) su misión es la siguiente:

...supervisar las auditorías de las empresas públicas con el fin de proteger los intereses de los inversores y promover el interés público en la preparación de informes de auditoría informativos, precisos e independientes. EI PCAOB también supervisa las auditorías de los corredores de bolsa, incluyendo los informes de cumplimiento presentados de conformidad con las leyes federales de valores, para promover la protección de los inversores (PCAOB, 2016b).

EI PCAOB marca las directrices bajo las cuales los auditores deben realizar su trabajo, con el objetivo de dar verdadera seguridad a los inversionistas que ponen su confianza en las opiniones profesionales que ellos emiten. Dichas normas marcan principios mínimos y básicos que aseguran la calidad de la auditoria que ellos realizan.

La Ley Sarbanes-Oxley establece en su sección 404 (Senate and House of Representatives of the United States of America in Congress assembled, 2002), la obligación de que las empresas que coticen en la bolsa de valores cuenten con un informe de evaluación de su sistema de control interno, emitido por un auditor externo. Es aquí donde el PCAOB establece en su normatividad, en el estándar 2201, la manera en que debe realizarse este tipo de revisiones (PCAOB, 2016a). 


\section{Definición de auditorías integradas}

Debido a que la Ley SOX obliga a las compañías públicas a que un auditor externo independiente emita una opinión acerca de su control interno, los auditores necesitan realizar una "auditoría integrada" para poder emitir su informe. Ya que este artículo es referente a los procedimientos de auditoría que harán más eficientes las revisiones efectuadas en auditorías integradas, es importante definir correctamente este punto.

De acuerdo a Marin Calvo (2013), podemos definir que auditoría financiera

...es aquel proceso cuyo resultado final es la emisión de un informe, en el que el auditor da a conocer su opinión sobre la situación financiera de la empresa, este proceso solo es posible llevarlo a cabo a través de un elemento llamado evidencia de auditoría, ya que el auditor hace su trabajo posterior a las operaciones de la empresa.

En contraste con la auditoría operativa que Marin Calvo (2013) define como:

...el examen posterior, profesional, objetivo y sistemático de la totalidad o parte de las operaciones 0 actividades de una entidad, proyecto, programa, inversión o contrato en particular, sus unidades integrantes u operacionales específicas. Su propósito es determinar los grados de efectividad, economía y eficiencia alcanzados por la organización y formular recomendaciones para mejorar las operaciones evaluadas. Relacionada básicamente con los objetivos de eficacia, eficiencia y economía.

Según Mauro Vera (2014), una auditoría integrada es aquella que combina pasos de una auditoría financiera y una operativa (ver Figura 1), es decir, no solo se busca cubrir el aspecto financiero de la entidad, sino también el control interno de la misma, de acuerdo a toda la nueva normatividad establecida por el PCAOB (PCAOB, 2016a). La gran cantidad de requisitos que establece el PCAOB y el detalle de los mismos para realizar este tipo de auditorías, ha traído una gran carga de trabajo a los auditores en comparación con periodos anteriores a la entrada en vigencia de esta ley.

Es por ello que las firmas de auditoría y auditores independientes buscan eficientar cada vez más sus procesos de revisión para lograr un alcance adecuado en sus pruebas, pero sin invertir una cantidad excesiva de tiempo en las mis- 
mas; para lograrlo requieren conocer a detalle todos estos cambios ahora existentes y buscar entender cuál es el objetivo general para este tipo de revisiones.

Figura 1. Diferencias entre la auditoría financiera y la auditoría integrada

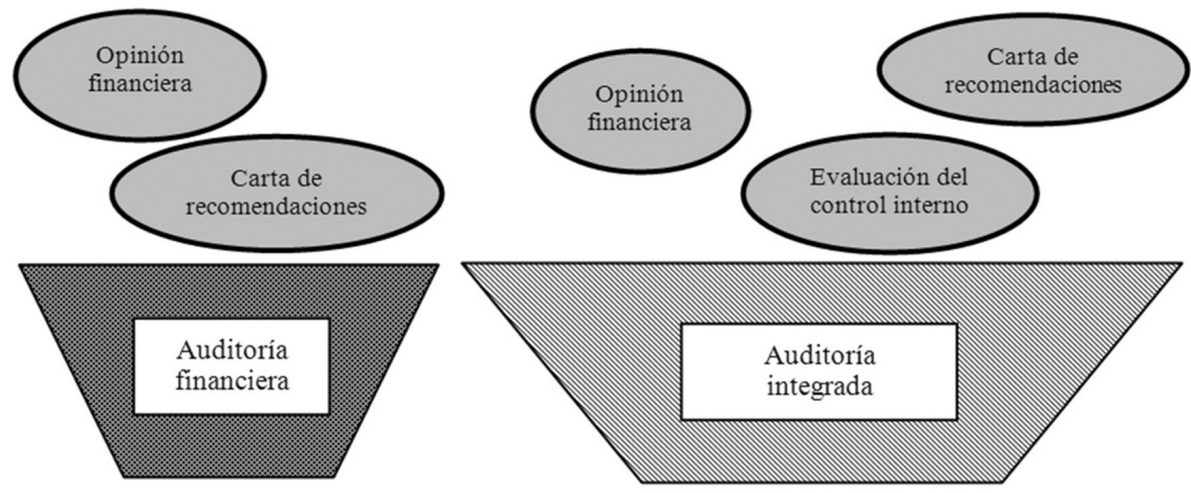

Fuente: Elaboración propia basada en presentación de Mauro Vera (2014)

\section{Definición de control interno}

Ya que este artículo va enfocado a las auditorías integradas, es decir, aquellas donde se emite una opinión del control interno, es importante definir correctamente este término para que el auditor comprenda el objetivo que debe conseguir cuando realice este tipo de auditorías.

Se entiende por control interno a todas aquellas políticas y procedimientos que la compañía implementa y da seguimiento para:

a) obtener información financiera confiable;

b) salvaguardar adecuadamente sus activos;

c) eficientar sus operaciones

En una auditoría integrada, el principal enfoque es la revisión de aquellos controles que intervienen e impactan de algún modo la información financiera que se reporta.

Un conocimiento amplio y claro del control interno es lo que le permitirá al auditor aplicar correctamente sus procedimientos de auditoría. La identificación correcta de los controles clave de la compañía propiciará la eficiencia en el trabajo que el auditor desempeñe. La revisión del control interno está sujeto a un marco normativo específico, establecido por el PCAOB, que es conocido como 
Marco de Referencia COSO 2013 (Committe of Sponsoring Organizations of the Treadway Commission, 2016).

\section{Normas referentes al control interno para empresas públicas}

Después de todos los escándalos ya mencionados, la SEC decidió tomar acciones claras y contundentes para prevenir futuros incidentes similares. Además de la creación de la Ley Sarbanes-Oxley y del PCAOB, se estableció uno de los principales cambios que robusteció las medidas ya establecidas. Los principales ejes de cambio se abocaron a endurecer los sistemas de control interno sobre la información financiera que deben de mantener las empresas emisoras.

Así la Ley Sarbanes-Oxley estableció, entre otras, estas dos obligaciones para las emisoras (Buzo, 2014):

1. Las administraciones deben de autoevaluar y opinar sobre la efectividad de su control interno (sección 302, Senate and House of Representatives of the United States of America in Congress assembled, 2002).

2. El auditor externo debe de emitir un dictamen sobre la efectividad del control interno como base para la generación de la información financiera de la misma entidad (sección 404, Senate and House of Representatives of the United States of America in Congress assembled, 2002).

Así como para el punto número anterior, los auditores externos determinan un marco de referencia bajo el cual emitirán su opinión sobre los estados financieros de sus clientes como, por ejemplo, Normas Internacionales de Información Financiera (IFRS por sus siglas en inglés), Normas de Información Financieras Mexicanas (NIF por sus siglas en español), Principios de Contabilidad Estadounidenses (USGAAP por sus siglas en inglés), etc; la SEC y el PCAOB determinaron el marco de referencia COSO para todas las opiniones que se realicen sobre el control interno de las compañías públicas.

COSO, organizado en 1985, fue patrocinado por la Comisión Nacional de Informes Financieros Fraudulentos, una iniciativa independiente del sector privado que estudió los factores causales que pueden conducir a informes financieros fraudulentos. También desarrolló recomendaciones para las empresas públicas y sus auditores independientes, para la SEC y otros reguladores, y para las instituciones educativas (Committe of Sponsoring Organizations of the Treadway Commission, 2016). La principal misión de COSO es: 
Proporcionar liderazgo intelectual a través del desarrollo de marcos generales y orientaciones sobre la Gestión del Riesgo, Control Interno y Disuasión del Fraude, diseñado para mejorar el desempeño organizacional y reducir el alcance del fraude en las organizaciones (Committe of Sponsoring Organizations of the Treadway Commission, 2016).

\section{Resultados}

Al entender la normatividad expuesta en los puntos anteriores y los objetivos clave que las auditorías integradas persiguen, es necesario que los auditores diseñen y evalúen cuáles son las estrategias que seguirán para cumplir con esta clase de compromisos.

El Comité Internacional de Normas de Auditoría y Aseguramiento (IAASB, por sus siglas en inglés, International Auditing and Assurance Standards Board) es una entidad independiente cuyo principal fin es emitir estándares y normas de auditoría de alta calidad (International Federation of Accountants [IFAC], 2016). Aunque para el caso de auditorías bajo normas PCAOB, dichos procedimientos deben ser revisados para evaluar si el alcance, naturaleza y oportunidad de los procedimientos son los adecuados para cumplir con los estándares establecidos (PCAOB, 2016a).

Para las compañías, la aplicación de toda la normatividad establecida por la ley SOX, resulta sumamente costosa, y para algunos aún más en relación con los beneficios que se obtienen (Grundfest \& Bochner, 2007). Es aquí donde focalizar correctamente los procedimientos ayudará a disminuir el tiempos y los costos para las firmas de auditoría, ya que solo se invertirá tiempo en los puntos esenciales y, por ende, tendrán un mejor margen para negociar el precio de los servicios prestados con sus clientes. De acuerdo a Premuroso \& Houmes (2012)

...realizar una evaluación del riesgo de los estados financieros es una habilidad vital para ayudar a asegurar tanto el cumplimiento por parte de los auditores como de las empresas públicas de las directrices contenidas en la Ley Sarbanes-Oxley de 2002 (SOX), la Guía Interpretativa de la SEC, relativa al Control Interno de la Información Financiera, el marco de evaluación de deficiencias de control que se encuentra en la Norma de Auditoría No. 5 (AS5) del Consejo de Supervisión de Contabilidad de Empresas Públicas (PCAOB) y el Comité de Organizaciones Patrocinadoras de la Comisión Treadway (COSO). 
Es decir, una correcta evaluación de riesgos y una correcta aplicación de procedimientos de auditoría que mitiguen dichos riesgos detectados no solo permitirá ver un beneficio desde el punto de vista de los costos incurridos, sino que también permitirá aplicar correctamente los procedimientos de auditoría en las áreas significativas, para obtener una seguridad razonable de que no existen errores de error material en las cifras revisadas, y verdaderamente ser eficientes en el trabajo que se realiza (Premuroso \& Houmes, 2012).

Los resultados de este artículo no pretenden solucionar problemáticas de revisiones en rubros específicos de los estados financieros, ni tampoco centrarse en áreas específicas de una compañía, sino por el contrario, pretende enfocar su atención a algunas estrategias que pudieran aplicarse a varios ámbitos 0 áreas de las revisiones de auditoría, de acuerdo a los estándares oficiales publicados por el PCAOB. Con base en la investigación realizada acerca de estrategias y mejores procedimientos a aplicar para eficientar el trabajo de auditoría se pretender abarcar tres puntos específicos:

1. Mejor entendimiento de la entidad: sus procesos y su control interno.

2. Mayor utilización del trabajo del departamento de auditoría interna de la compañía.

3. Mayor aplicación de procedimientos analíticos como herramienta en la identificación y evaluación de riesgos, y como procedimientos sustantivos a aplicar.

Cada uno de esos puntos se desglosará a continuación a lo largo de este artículo de investigación, y su aplicación pretende eficientar los costos y tiempos del trabajo a realizar:

1. Mejor entendimiento de la entidad: sus procesos y su control interno.

De acuerdo con Brown (2012), la experiencia y el conocimiento que tienen los auditores de una compañía o de un sector les da la oportunidad de disminuir los costos de producción (costos de realización de sus servicios) debido al conocimiento adquirido y acumulado de la entidad.

Brown (2012) resalta que aprovechar este conocimiento le facilitará al auditor especializarse en algún tipo de empresa, lo que le permitirá una mayor eficiencia en la auditoría y además logrará un menor riesgo al auditar debido a que aplicará los procedimientos de auditoría donde se conoce que pudieran existir errores materiales. 
De acuerdo con Chen, Gul, Truong \& Veeraraghavan (2016), existe una relación directa positiva entre la cercanía del trato del auditor y el conocimiento que tiene de sus clientes por el largo tiempo de conocerse y trabajar con ellos, dicha relación disminuye el número de debilidades en el control interno encontradas en tales compañías (ICW, por sus siglas en inglés que significan Internal Control Weakness).

Se ha comprobado que existen mayores debilidades en el control interno de las compañías cuando sus auditores no son tan cercanos con sus clientes 0 , en su defecto, no cuentan con el conocimiento suficiente de los mismos ya sea porque son sus auditores por primera vez en el año o cuando dichos auditores no logran entender de manera correcta los procesos y controles de sus clientes.

Existen procedimientos clave que los auditores pueden utilizar para entender y documentar de mejor manera los procesos y los controles, y que facilitarán la identificación de las áreas clave donde el auditor deberá enfocar sus pruebas para eficientar sus revisiones. Algunos de estos procedimientos son:

- Realizar pruebas de recorrido o walkthroughs. Consisten en dar seguimiento a las transacciones de los ciclos considerados como significativos desde su origen hasta la conclusión de las transacciones, y su impacto en los estados financieros (Miralrios en Prezi, 2013).

- Documentación de diagramas de flujo de transacciones o flowcharts. Este tipo de herramientas permiten al auditor identificar gráficamente cuál es el ciclo, proceso y forma en que fluye la información a través de toda una transacción y, por ende, detectar cuáles son los posibles riesgos que podrían detonarse para determinados casos. Por este medio gráfico es más sencillo identificar también cuáles son los controles que tiene la compañía a lo largo de dicha transacción y cuáles serían los que, para efecto de auditoría, serían relevantes para probar su desempeño en la auditoría integrada (KPMG, 2014).

Este tipo de pruebas sirven para cumplir dos grandes objetivos: conocer mejor los procesos y transacciones de las compañías de inicio a fin para efecto de entender mejor sus operaciones y detectar las áreas donde hay que enfocar la atención para revisar y entender mejor su control interno. En este proceso, para efectos del PCAOB, se tiene que evaluar en revisiones de control interno cuando se realizan auditorías integradas de acuerdo a los estándares AS 2110 y AS 2201 (PCAOB, 2016a). 
Estas pruebas, junto con otras de la misma naturaleza, le permitirán al auditor eficientar los recursos y tiempo empleados en la revisión ya que solo se enfocará en aquello que, a su juicio, son transacciones/controles significativos, y no perderá el tiempo ni recursos en aquello en que de acuerdo a su conocimiento no es relevante, mejorando así la eficiencia de su trabajo.

\section{Mayor utilización del trabajo del departamento de auditoría interna de la compañía.}

De acuerdo a la sección 302-4A de la Ley Sarbanes-Oxley (Senate and House of Representatives of the United States of America in Congress assembled, 2002) las empresas y su administración son las responsables de:

- Establecer y mantener el control interno de la compañía.

- Diseñar controles para asegurarse que la información significativa relacionada con la emisora y sus subsidiarias consolidadas sea conocida a tales funcionarios por otros dentro de esas entidades, particularmente durante el período en que se preparan los informes periódicos.

- Evaluar la efectividad de los controles internos del emisor a partir de una fecha dentro de los 90 días anteriores al informe.

- Presentar en el informe sus conclusiones sobre la eficacia de sus controles internos sobre la base de su evaluación a partir de esa fecha.

Para dar cumplimiento a estas disposiciones las compañías tienen que crear departamentos de auditoría interna para que les ayuden a evaluar estos puntos. En el caso de las compañías que tienen varios años como emisoras en la bolsa de valores, es común que cuenten con un departamento de auditoría interna muy robusto debido a que, al paso de los años, han ido mejorando sus procesos de revisión o diseño de controles y llevan un trabajo de alta calidad en cuanto a la evaluación interna de su compañía.

Entre mejor establecido esté el departamento de auditoría interna de una compañía y entre más profesional sea el trabajo que ejecuten en la evaluación, diseño e implementación de controles internos, más confiable será la información que reporten y es menos probable encontrar deficiencias significativas que afecten la confianza en la misma, ya que el mismo departamento prevendrá, detectará y corregirá las deficiencias en tiempo y forma, antes de que impacten de manera definitiva los estados financieros de la entidad. 
Es en estos casos, cuando la entidad tiene un departamento de auditoría interna de tal calidad, que los auditores externos pueden utilizar y confiar en cierta medida en el trabajo realizado por ellos y concluir de manera expedita su revisión en auditorías integradas (Malaescu \& Sutton, 2015).

De acuerdo con Felix, Gramling, \& Maletta (2005) y Felix, Gramling, \& Maletta (2001), citados en Munro \& Stewart (2009): "En la medida en que el auditor externo se base en el trabajo de auditoría interna, es una decisión clave de planificación de la auditoría que puede tener un impacto significativo en los honorarios de auditoría".

Todo ello debido a que el alcance de la revisión sería meno al tener una fuente confiable de revisión y, por ende, menores costos de personal invertidos en la auditoría. El PCAOB en su estándar número AS 2605 (PCAOB, 2016a), establece los criterios que deben de tomar en cuenta los auditores externos, para evaluar la calidad de trabajo realizado, entre los cuales se encuentran:

- Entender las funciones y labores del departamento de auditoría interna.

- Evaluar la competencia, objetividad e independencia de los miembros del departamento de auditoría interna relacionados a las revisiones.

- Entender el alcance, naturaleza y oportunidad de los procedimientos de revisión del departamento de auditoría interna.

- Revisar el trabajo realizado por el departamento de auditoría interna, entre otros.

Cuando el auditor externo evalúa de acuerdo al AS 2605 (PCAOB, 2016a) y constata que el departamento de auditoría interna es competente, es cuando pude optar, de acuerdo a su planeación, utilizar o no información generada y revisada por ellos para concluir y emitir una opinión acerca del control interno de la compañía.

Utilizar la información revisada por auditoría interna ayudará a mejorar el entendimiento de los procesos y transacciones de la compañía al entender cómo enfocan sus revisiones, este proceso disminuirá los costos y el tiempo del personal asignado debido a que habrá un menor alcance de la revisión, ya que gran parte de la documentación partirá de la función de auditoría interna.

De acuerdo a Glover, Prawitt, \& Wood (2008), citado por Munro \& Stewart (2009): "Los beneficios económicos de la confianza de auditores externos en trabajo de auditoría interna son bien conocidos". De acuerdo a Felix et al. (2001) 
citado por Munro \& Stewart (2009), los honorarios de auditoría disminuyen aproximadamente un $18 \%$, cuando los auditores externos coordinan su trabajo con los auditores internos.

\section{Mayor aplicación de procedimientos analíticos como herramienta}

en la identificación y evaluación de riesgos y como procedimientos sustantivos a aplicar

La tercera estrategia por abordar es el uso/aplicación de procedimientos analíticos tanto sustantivos como preliminares de evaluación. Se entiende por procedimientos analíticos aquellos que "...constituyen el análisis de índices y tendencias, incluyendo la investigación de fluctuaciones y relaciones que son inconsistentes con otra información relevante o que se desvían de las cantidades presupuestadas" (Keller Kaplanska, 2006).

En contraste con los procedimientos de revisión sustantivos convencionales que son aquellos que se enfocan "...en particular en la revisión documental, mediante pruebas de detalle de las principales partidas y elementos que forman los estados financieros" (Sotomayor Gonzalez, 2010).

Entendiendo por lo anterior que los procedimientos analíticos permitirán al auditor entender, desde una perspectiva global, la operación de la compañía, lo que le permitirá eficientar sus procedimientos de auditoría desde la planeación, la ejecución y su conclusión (Keller Kaplanska, 2006; Sotomayor González, 2010).

En la planeación de auditoría, estos procedimientos son aplicables para entender las variaciones entre períodos, tendencias, saldos reales vs. presupuestos, etc.; para entender en qué rubros o saldos de cuenta enfocar el trabajo debido a dichos cambios/variaciones entre periodos; para que, de igual manera que los procedimientos, conocer la entidad (estrategia número 1, antes comentada), enfocar esfuerzos donde realmente sea significativo y eficientar el trabajo que se realizará. De acuerdo a Sotomayor González (2010),

...La utilización de los procedimientos analíticos permite al auditor, de una manera eficiente, identificar posibles riesgos de error, pero no sólo esto, sino que también le permite conocer sobre la entidad auditada, su desarrollo financiero y las tendencias de la industria de la entidad. 
Durante la ejecución del trabajo de auditoría los procedimientos analíticos le permiten al auditor obtener evidencia suficiente y adecuada para concluir sobre algún saldo de cuenta o transacción, sin la necesidad muchas veces de entrar en revisión de detalles como pruebas sustantivas comunes. Este tipo de transacciones son útilmente aplicables a grandes volúmenes de transacciones que tienden a ser predecibles en el tiempo (Sotomayor González, 2010), donde el análisis, comparación e interpretación de las variaciones será suficiente para obtener conclusiones debidamente soportadas acerca de algún saldo a revisar, sin la necesidad del detalle y tiempo invertido que se incurre en otros procedimientos de revisión.

Durante la conclusión de auditoría, estos procedimientos son sumamente útiles para entender el cierre de los ciclos y su reflejo en los estados financieros mediante el análisis de razones financieras, variaciones significativas, tendencias, etc. Estos procedimientos de auditoría le permitirán concluir al auditor su informe de auditoría con suficiente evidencia de que su revisión se efectuó considerando todos los puntos significativos de la compañía, ya que le permitirán tener un mejor panorama del comportamiento de la compañía. De acuerdo con Sotomayor González (2010),

"...se considera que el auditor debe promover el uso de procedimientos analíticos para efectos de hacer eficiente su práctica profesional, buscando la reducción de tiempos y el logro de objetivos de auditoría de una manera más eficaz y sólida".

\section{Discusión}

La manera en que los auditores desempañan su trabajo profesional ha cambiado significativamente en los últimos años. Escándalos financieros de empresas reconocidas en mercados de valores internaciones han propiciado que los entes financieros reguladores establezcan más requisitos y normas para el ejercicio laboral de estos profesionales, con el objetivo de generar mayor confianza en los informes independientes que éstos emiten para efecto de aquellas entidades que coticen en bolsa de valores.

Las firmas de auditoría, impulsadas por la urgencia de cumplir con cada vez más estrictos estatutos estipulados por dichos reguladores, buscan la manera de eficientar sus trabajos de revisión, sin descuidar la calidad requerida de los 
mismos. Es por ello el interés cada vez mayor de aplicar estrategias y mejores prácticas de auditoría para cumplir con el alcance de revisión requerido por las autoridades correspondientes, sin que esto afecte el presupuesto de auditoría para sus clientes. Existen muchos procedimientos y mejores prácticas útiles para cumplir este propósito, pero los siguientes son algunos que eficientarán el tiempo y los costos incurridos en auditorías integradas:

1. Mayor aplicación de procedimientos de entendimiento y conocimiento de la entidad, que le permitirán al auditor aplicar procedimientos de auditoría solo donde después de su revisión, y de acuerdo a su juicio profesional, se detecten áreas significativas, evitando invertir tiempo y esfuerzo sobre rubros que no son importantes.

2. Evaluación y utilización del trabajo de auditoría interna para efecto de aprovechar su documentación y disminuir el alcance de la revisión del auditor, permitiendo que se invierta un menor tiempo y recursos en personal para la ejecución de auditoría.

3. Mayor aplicación de procedimientos de revisión analíticos que le permitirán al auditor tener un mejor entendimiento de la compañía, para efecto de aplicar mejor los procedimientos de auditoría sobre saldos de cuenta o transacciones identificados como materiales, que le permitirán obtener evidencia suficiente y adecuada para concluir su revisión sin la necesidad, la mayoría de las veces, de aplicar pruebas de detalle que conllevan en mayor tiempo para la revisión y documentación de las mismas.

Todos estos procedimientos deben de ser evaluados por el equipo de auditoría de cada compromiso para efecto de entender de qué manera aplicarlos en su trabajo, confiando que todo ello ayudará a ser más eficientes, buscando disminuir los costos y tiempo invertidos, pero sin descuidar la calidad y confianza en la opinión que dichas firmas de auditoría emiten. 


\section{Referencias}

Beckstead, N. (2002). The Sarbanes-Oxley Act: Investors Protection through Corporate Governance. Brigham Young University, 18.

Brickey, K. F. (2003). From Enron to Worldcom and Beyond: Life and Crime After SarbanesOxley. Washington University Law Review, 81(2), 357-401.

Brown, S. V. (2012). Specialization Through Client Commonality and Its Effect on Audit Production Cost. Fisher School of Accounting University of Florida, 49, 1-15.

Buzo, J. (2014). Punto de vista. 2014, año de trancisión al nuevo COSO 2013 PricewaterhoseCoopers, S.C. (PwC México), en www.pwc.com/mx

Chen, Y., Gul, F. A., Truong, C., \& Veeraraghavan, M. (2016). Auditor client specific knowledge and internal control weakness: Some evidence on the role of auditor tenure and geographic distance. Journal of Contemporary Accounting and Economics, 12(2), 121-140.

Committe of Sponsoring Organizations of the Treadway Commission. (2016). Committee of Sponsoring Organizations of the Treadway Commission: About us, en http://www.coso.org/aboutus.htm

Cortijo Gallego, Vi. (2007). Impacto de la Ley Sarbanes-Oxley en la regulación del sistema financiero español. Boletín ICE Económico, 2907, 43-51.

Díaz Morales, J. (2005). La Ley Sarbanes-Oxley y la auditoría. Partida Doble, 169, 104-109. Felix, W. L., Gramling, A. A., \& Maletta, M. J. (2001). The contribution of internal audit as a determinant of external audit fees and factors influencing this contribution. Journal of Accounting Research, 39(3), 513-534.

Felix, W. L., Gramling, A. A., \& Maletta, M. J. (2005). The influence of nonaudit service revenues and client pressure on external auditor's decisions to rely on internal audit. Contemporary Accounting Research, 22(1), 31-53.

Fornelli, C. M. (2015). The creation of the PCAOB: A watershed moment. The CPA Journal, 85(4), 6-8.

Glover, S. M., Prawitt, D. F., \& Wood, D. A. (2008). Internal audit sourcing arrangement and the external auditor's reliance decision. Contemporary Accounting Research, 83(2), $447-478$.

Grundfest, J. A., \& Bochner, S. E. (2007). Fixing 404. Michigan Law Review, 105 (8), 1643-1676. International Federation of Accountants (IFAC) (2016). The International Auditing and Assurance Standards Board (IAASB), en https://www.iaasb.org/

Keller, V. (2006). Procedimientos analíticos de revisión. Comisión de Auditoría, Instituto Mexicano de Contadores Públicos, Boletín del Colegio de Contadores Públicos de México, 16(1), 1-4.

KPMG. (2014). Better understanding the process through flowcharting An implementation guide, KPMG Executive Education: Montvale, NJ.

$\mathrm{Li}$, Y. (2010). The Case Analysis of the Scandal of Enron. International Journal of Business \& Management, 5(10), 37-41. 
Malaescu, I., \& Sutton, S. G. (2015). 1 The Reliance of External Auditors on Internal Audit's Use of Continuous Audit. Journal of Information Systems, 29(1), 95-114.

Marín Calvo, H. A. (2013). Auditoría financiera, en http://www.gerencie.com/auditoriafinanciera.html

Mauro Vera, J. L. (2014). Preparándose para una auditoría integrada. Congreso Internacional sobre Gobierno, Riesgos, Auditoría y Seguridad de Información (CIGRAS).

Miralrios en Prezi, S. M. en. (2013). Pruebas de recorrido, en https://prezi.com/6xo4d4005u4o/ pruebas-de-recorrido/

Munro, L., \& Stewart, J. (2009). External auditor's reliance on internal audit: the impact of sourcing arrangements and consulting activities. Griffith University, 52(3), 202-205.

Nagy, D. M. (2005). Playing peekaboo with constitutional law: The PCAOB and its public/ private status. Notre Dame Law Review, 80(3), 975-1071.

PCAOB. (2016a). PCAOB Auditing Standards, en https://pcaobus.org/Standards/Auditing/ Pages/ReorgStandards.aspx

PCAOB. (2016b). Public Company Accounting Oversight Board (PCAOB), en https://pcaobus. org/Pages/default.aspx

Premuroso, R. F., \& Houmes, R. (2012). Financial statement risk assessment following the COSO framework. International Journal of Accounting \& Information Management, 20(1), 26-48.

Reiter, S. A., \& Williams, P. (2013). Sarbanes-Oxley and the Accounting Profession: Public Interest Implications. Open Journal of Accounting, 2(1), 8-15.

Senate and House of Representatives of the United States of America in Congress assembled. (2002). Sarbanes-Oxley Act of 2002. Public Law 107-204; 107th Congress, en http://www2.ed.gov/policy/elsec/leg/esea02/107-110.pdf

Sotomayor González, R. (2010). Procedimientos analíticos en las auditorías de estados financieros. Revista Contaduría Pública: Instituto Mexicano de Contadores Públicos, 1-5.

WebFinance Inc. (2016). What is Public Company? definition and meaning, en http://www.investorwords.com/3932/public_company.html 